•综述・

\title{
风媒传粉的研究方法探讨
}

\author{
朱亚如 龚燕兵* \\ (武汉大学生命科学学院杂交水稻国家重点实验室 武汉 430072)
}

\begin{abstract}
摘要: 种子植物的花粉传递依赖多样化的传粉媒介, 与动物传粉相比, 风媒传粉往往被认为效率较低而受到较少 关注。然而, 绝大部分裸子植物和至少 $10 \%$ 的被子植物依赖风媒传粉, 在被子植物中至少发生了 65 次从动物媒向 风媒的传粉转变, 而且同种植物内风媒与虫媒并存的混合传粉机制也已屡见报道。因此, 函需更加完善的研究方 法以揭示生态系统中大量隐藏的风媒传粉现象和机理。本文首先介绍了风媒传粉植物的种类和生境多样性, 以明 确风媒传粉的研究范围。在此基础上, 论述了在野外条件下应首选使用花粉捕获法(黏性玻片或空气颗粒取样器) 测量空气中的花粉数量; 同时应结合传粉者排除法和套袋、去雄等传粉处理, 确定风媒传粉的生殖贡献大小以及 动物传粉、自动自交和无融合生殖并存的可能性。如果野外条件受限, 应采集相关植物组织, 在实验室使用频闪 摄影或沉降塔法测量花粉的沉降系数以推测风媒传粉的可能性, 并利用风洞实验和计算机模拟探明不同条件下的 风媒传粉效率和空气动力学基础。同时, 应进一步研究影响风媒传粉的生物(风媒传粉综合征)和非生物因素, 以探 寻风媒传粉发生的原因和进化生态后果。以上方法并不能相互替代, 研究者应尽量全面使用各类方法以准确描述 风媒传粉现象并解释其适应机理。
\end{abstract}

关键词: 风媒传粉; 种子植物; 花粉; 野外方法; 空气动力学

\section{Methods of wind pollination}

Yaru Zhu, Yanbing Gong*

State Key Laboratory of Hybrid Rice, College of Life Sciences, Wuhan University, Wuhan 430072

\begin{abstract}
The transfer of pollen in most seed plants relies on diverse pollination vectors. In comparison with animal pollination (zoophily), wind pollination (anemophily) has long been regarded as an inefficient mode and thus has received relatively little attention. However, the majority of gymnosperm species and over $10 \%$ of angiosperm species are wind pollinated, and the evolution of wind pollination from insect-pollinated ancestors has occurred at least 65 times in angiosperms. Furthermore, ambophily, a combination of wind and insect pollination, is also reported frequently. More refined methods are thus seriously needed to explore the existence and mechanisms of wind pollination in diverse ecosystems. In this paper, we explore the scope of anemophily research by describing the species and habitat diversities of wind-pollinated plants. In field experiments, we recommend using pollen traps (sticky slides or airborne particle samplers) to quantify airborne pollen, and conducting pollinator exclusion, bagging, and emasculation treatments to explore the reproductive contribution of anemophily and the possibilities of zoophily, autogamy, and apomixis. In constrained field conditions, researchers can bring relevant plant tissues back to the laboratory for experiments examining aerodynamics, i.e., measuring the settling velocity of pollen using stroboscopic photography or drop towers, calculating the pollination efficiency using wind tunnels, and evaluating the aerodynamics based on computer models in different simulated conditions. Furthermore, the abiotic and biotic factors (wind pollination syndromes) associated with anemophily should also be studied to explore the causes as well as the ecological and evolutionary consequences of wind pollination. The above methods cannot substitute for one another, as researchers should use them as a comprehensive unit when possible to reveal the details and mechanisms of wind pollination.
\end{abstract}

Key words: wind pollination; seed plants; pollen; field methods; aerodynamics

收稿日期: 2017-03-06; 接受日期: 2017-05-04

基金项目: 国家自然科学基金(31670228)和湖北省自然科学基金(2011CDA095)

* 通讯作者 Author for correspondence. E-mail: ybgong@whu.edu.cn 
种子植物的花粉传递依赖不同的传粉媒介，虫 媒传粉的多样性、普遍性和高效性使其受到大量关 注(黄双全和郭友好, 2000), 而水媒和风媒传粉往往 被认为效率较低而在自然生态系统中处于劣势地 位(Darwin, 1876; Faegri \& van der Pijl, 1979)。然而, 新的研究表明, 风媒传粉并不如想象中那么低效, 在某些环境条件或某些植物类群中, 其效率甚至高 于虫媒传粉(Friedman \& Barrett, 2009)。目前, 被子 植物中已经发现至少 65 次从动物媒向风媒的传粉 转变(Linder, 1998; Friedman, 2011)。传粉模式从虫 媒转变为风媒, 相对普遍的原因可能在于植物只需 要进化出相对简单的花部特征就可以适应风媒; 但 从风媒转变为虫媒在自然界中却很少存在, 可能因 为植物要重新获得与吸引、报酬和适应昆虫访花行 为相关的复杂花部特征, 会经历更大的进化限制 (Huang et al, 2013)。因此, 通常认为从虫媒到风媒 的传粉系统转变在某种程度上是不可逆的 $(\mathrm{Cox}$, 1991; Dodd et al, 1999)。按照以上观点, 随着全球自 然环境和动物传粉服务水平的双重变化(Hegland et al, 2009; Ollerton et al, 2014), 植物界中将极可能出 现更高比例和多样化的风媒传粉现象。

传粉生物学研究中对风媒贡献的忽略往往源 自对研究方法理解和运用上的一些误区。如某些植 物被观察到了昆虫访问, 抑或其具有某些适应虫媒 传粉的特征, 如散发气味、具有鲜艳颜色等, 都被 认为是虫媒传粉的; 但最新研究表明, 具以上花部 特征的如买麻藤属 (Gnetum) 和乌头属(Aconitum) 植 物, 均存在风媒传粉模式(Gong et al, 2016; Wang et al, 2016)。此外, 与虫媒传粉研究相似, 短时的观察 和片面的实验并不能得到准确全面的结论(共燕兵 和黄双全, 2007); 如静风条件下的风媒传粉实验, 往往会错误评估植物的风媒传粉效率 (Niklas, 2015)。这些研究方法上的缺陷, 极可能导致对植物 风媒传粉存在性的不正确描述, 从而严重低估自然 界中风媒传粉的普遍性和多样化的风媒传粉策略 (Owens et al, 1998)。基于以上背景, 本文总结并评 价了风媒传粉的研究方法, 以期能对开展相关植物 繁殖生态和保护研究的学者提供方法学上的参考。

\section{1 风媒传粉植物的多样性}

\section{1 种类多样性}

种子植物在不同选择压力下进化出多样化的
传粉模式, 其中虫媒传粉植物的普遍性已受到大量 关注，而风媒传粉植物的种类多样性往往被低估。现 存五大类种子植物——银杏(Ginkgo biloba)、松柏类 (conifers)、苏铁类 (cycads)、买麻藤目和被子植物中 (Wang \& Ran, 2014), 都已先后报道了不同比例风媒 传粉模式的存在(Ackerman, 2000; Cully et al, 2002)。

裸子植物被认为是风媒传粉起源的, 其中银杏 和松柏类植物都是风媒传粉的(Owens et al, 1998; Jin et al, 2012); 苏铁类植物中虫媒和风媒传粉均能 发挥作用(王乾等, 1997; Kono \& Tobe, 2007); 买麻 藤目中风媒是最主要的传粉方式, 但在各属中比例 有所不同(Ickert-Bond \& Renner, 2016)。麻黄属 (Ephedra) 以风媒传粉为主(Bolinder et al, 2015), 而 百岁兰 (Welwitschia mirabilis) 则主要为虫媒传粉 (Wetschnig \& Depisch, 1999), 早期研究认为买麻藤 属是专性虫媒传粉的(Kato \& Inoue, 1994), 但最新 研究却揭示了其风媒传粉的存在(Gong et al, 2016)。

对于虫媒传粉起源的被子植物, 风媒传粉被认 为是从虫媒传粉转变而来的衍生模式, 业已发现被 子植物的许多类群中风媒传粉是独立进化而来的 (Friedman \& Barrett, 2009)。Ackerman (2000)的系统 调查显示, 风媒传粉的被子植物分布在木兰亚纲、 金缕梅亚纲、石竹亚纲、五桠果亚纲、蓄薇亚纲、 菊亚纲、泽泻亚纲、棕榈亚纲、鸭跖草亚纲和百合 亚纲中。在目的水平，除鸭跖草亚纲中风媒传粉目 的比例高达 $85 \%$ 外, 其他各个亚纲都只有不到一半 的目中出现了风媒传粉; 而以每目中风媒传粉科的 数量做频率分布图, 则发现其符合对数正态分布规 律, 即绝大部分的目中仅有 $0-2$ 个科的植物是风媒 传粉的，比例最高的无患子目，有高达 5 个科的植 物具有风媒传粉模式。整体来讲, 被子植物中至少 有 $18 \%$ 的科和 $10 \%$ 的种是风媒传粉的 (Ackerman, 2000)。

\section{2 生境多样性}

风媒传粉植物在地球上并不是随机分布的, 而 是具有明显的地理和生态趋势(Whitehead，1969; Regal, 1982)。风媒花粉的成功传递不仅由风力决定, 许多生物与非生物因素都对风媒传粉有着重要影 响, 如不同生境中植物群落结构的多样性、动物传 粉服务的水平和稳定性、温湿度和降雨量等, 都能 显著影响该生境中风媒传粉植物的比例(详见表 1 和 下文第3节)。 
表1 风媒和虫媒传粉的主要影响因素比对

Table 1 Key factors associated with wind vs. animal pollination

\begin{tabular}{|c|c|c|}
\hline & 风媒传粉 Wind pollination & 动物传粉 Animal pollination \\
\hline \multicolumn{3}{|l|}{ 非生物因素 Abiotic factors } \\
\hline 分布 Distribution & 温带 Temperate & 热带或温带 Tropical or temperate \\
\hline 最佳风速 Optimum wind speed & 低至中等 Low to moderate & 零至低 Zero to low \\
\hline 降雨 Precipitation & 不频繁 Infrequent & 不频繁至频繁 Infrequent to common \\
\hline 湿度 Humidity & 低 Low & 中至高 Medium to high \\
\hline \multicolumn{3}{|l|}{ 生物因素 Biotic factors } \\
\hline 周围植被 Surrounding vegetation & 开阔 Open & 开阔至郁闭 Open to closed \\
\hline 同种密度 Conspecific density & 中至高 Moderate to high & 低至高 Low to high \\
\hline 开花一致性 Flowering consistency & 同步 Synchronous & 不同步 Less synchronous \\
\hline 开花数量 Flower number & 多 Many & 少 A few \\
\hline 花位置 Flower position & 离叶较远 Held away from vegetation & 无规律 Variable \\
\hline 花类型 Flower type & 单性 Unisexual & 两性 Hermaphrodite \\
\hline 花被 Perianth & 缺失或减小 Absent or reduced & 显眼 Showy \\
\hline 花颜色 Floral colour & 绿或白 Greenish or whitish & 艳丽 Constrasting \\
\hline 花气味 Floral scent & 无或退化 Absent or reduced & 常有 Often present \\
\hline 蜜腺 Nectaries & 缺失或减小 Absent or reduced & 具备 Present \\
\hline 柱头 Stigmas & 羽状 Feathery & 简单 Simple \\
\hline 单花胚珠数 Ovules per flower & 一或少 One or few & 多 Many \\
\hline 花粉量 Pollen grains number & 多 Many & 少 Few \\
\hline 花粉大小 Pollen size & $\begin{array}{l}\text { 变化较小, 一般10-50 } \mu \mathrm{m} \\
\text { Less variable, often 10-50 } \mu \mathrm{m}\end{array}$ & $\begin{array}{l}\text { 变化较大, 一般 }>60 \mu \mathrm{m} \\
\text { Highly variable, often }>60 \mu \mathrm{m}\end{array}$ \\
\hline 花粉黏性 Pollen viscosity & 干 Dry & 黏 Viscid \\
\hline 花粉表面纹饰 Pollen ornamentation & $\begin{array}{l}\text { 光滑, 花粉鞘缺失或退化 } \\
\text { Smooth with reduced/absent pollenkitt }\end{array}$ & $\begin{array}{l}\text { 复杂并具花粉鞘 } \\
\text { Often elaborate with pollenkitt }\end{array}$ \\
\hline
\end{tabular}

主要参考文献: Faegri \& van der Pijl, 1979; Culley et al, 2002; Friedman \& Barrett, 2009; 张大勇, 2004。

Key references: Faegri \& van der Pijl, 1979; Culley et al, 2002; Friedman \& Barrett, 2009; Zhang, 2004.

早期研究表明, 风媒传粉植物的比例往往随纬 度和海拔的上升具有明显增加的趋势 (Regal, 1982)。温带地区植物居群相对聚集并且遮挡较少, 因而有利于花粉在空气中传播并成功传递到同种 柱头。另外，温带地区一般都具有严格的季节性， 相对于热带地区其植物生长季节较短, 因此很多树 种通过早春开花以增加生长时间，同时可避免与盛 花期植物的传粉竞争。然而, 早春虫媒传粉服务相 对缺乏且不稳定, 使得这些植物中很多依赖风媒传 粉(Elzinga et al, 2007)。正因如此, 温带的落叶森林、 开阔草原、干旱荒漠及高山地区都易进化出风媒传 粉物种(如彭德力等, 2012)。Regal (1982)以北美木本 植物为对象, 统计了不同生境中风媒传粉植物的比 例, 发现不同群落间风媒传粉植物比例变化较大, 其中大部分温带森林中都有一半以上的种类是风 媒传粉的。
物种丰富的热带地区降雨较多、湿度较大、树 叶遮挡以及植物分散生长等都不利于风媒传粉，这 些因素在低地雨林中的影响尤为严重。然而, Vamosi 等(2006)的大范围调查显示, 这些高生物多样性地 区的植物因为虫媒传粉竞争而经历着更严重的花 粉限制; 而花粉限制的选择压可能介导植物即使在 这样不利的条件下也能进化出风媒传粉机制以寻 求生殖保障(Cully et al, 2002)。Bawa (1990)的早期 调查显示，热带低地雨林中也有 $1-4 \%$ 的物种具风 媒传粉模式。

\section{2 风媒传粉的研究方法}

\section{1 花粉捕获法}

探寻风媒传粉的可能性，可首先使用花粉捕获 装置(pollen traps)明确植株周围的空气中是否携有 花粉; 通过收集目标植物的花粉作为参照，根据花 
粉的大小、形状和表面纹饰等特征对捕获的花粉进 行计数(如Gong et al, 2016)。花粉捕获装置需悬挂于 植株附近的适当距离, 尽量分多个方向散开, 在每 个主方向上可设置距离梯度, 如此可量化风媒花粉 的传播能力。根据花期、散粉和柱头可授情况, 可 选择在白天或者晚上的合适时间段内悬挂装置。天 气干燥有风时更易捕获风媒花粉, 但在某些不利条 件如下雨或无风时使用该方法, 则可以对比评价不 同环境条件下的风媒传粉效率。

捕获花粉最简单有效因而被广泛使用的装置 是黏性载玻片(sticky slide), 即涂有凡士林、甘油或 其他任何黏性物质的显微镜载玻片。黏性玻片可以 通过多种形式安装以截获花粉, 如可平铺于培养皿、 玻璃筒等器血中, 再将器皿置于合适高度的标杆顶 端, 如此便可收集由于重力作用垂直下落的风媒花 粉; 也可将黏性玻片通过长尾夹和绳线垂直悬挂安 置, 以捕获因风力而横向运动的花粉; 抑或模拟目 标植物制作假花序, 作为底座大量安置玻片 (Ackerman \& Kevan, 2005)。崔大练等(2008)在北重 楼(Paris verticillata) 开花植株的东南西北 4 个方位 分别按照 $0.2 、 0.4 、 0.6$ 和 $0.8 \mathrm{~m}$ 的距离梯度安放涂有 凡士林的玻片, 并以2小时为间隔回收玻片统计花粉 捕获量, 从而得出该植物风媒花粉的传播能力和日 散布规律。

使用以上方法也可能面临一些问题, 如雨天时 玻片捕捉的花粉可能被雨水冲刷至黏性物质下层 而难以计数, 又或直接被冲刷掉影响结果准确性。 解决的办法是将玻片安放到顶端遮挡、但侧面敞开 的简易装置中(如Durham sampler, 详见Ackerman \& Kevan, 2005)。此外, 野外条件下携有花粉的昆虫可 能误碰玻片而影响实验结果。因此, 在显微计数时应 特别注意一些有明显触碰痕迹、花粉分布极不均匀 的玻片, 样本量充足时可考虑将其删除; 还可通过 观察确定玻片未被昆虫触碰、对玻片进行套网或在 昆虫不活跃期(如夜间)悬挂玻片(Dafni \& Dukas, 1986)等方法, 减少昆虫随机活动对风媒花粉捕获的 干扰。

黏性玻片只能对空气中的花粉密度及传播距 离进行相对定量测定, 精确测定需借助商业购买的 空气颗粒取样器, 如英国Burkard Scientific公司的 定量空气自动取样器和美国Sampling Technologies 公司的Rotorod取样器。这些仪器都能在单位时间内
吸取固定体积的空气从而实现花粉密度的精确测 定。前者在测定 $10 \mu \mathrm{m}$ 以下花粉时效率较高, 而后者 更适合测定大于 $10 \mu \mathrm{m}$ 的花粉(Frenz, 1999)。

\section{2 传粉者排除法}

传粉者排除法(pollinator exclusion)是验证风媒 传粉最直接有效的方法。一般来讲, 研究者需在植 物开花前使用合适孔径的网袋，套网隔绝潜在传粉 者但允许风媒花粉进入, 将套网组(风媒)与自然对 照组 (风媒+虫媒)的坐果率和结籽率进行比较, 便 能了解风媒传粉的生殖贡献情况(Kearns \& Inouye, 1993)。Culley等(2002)总结了使用该方法对风媒虫 媒混合传粉系统(ambophily)植物的研究, 发现不同 植物中, 风媒的传粉贡献比例变化较大 (8-93\%), 但大部分处于中间水平。在实验过程中, 以下三类 因素的影响可能导致实验结果不理想或难以解释, 需在野外引起重视。

其一为网袋因素。一般的网袋都空间有限, 在 野外使用过程中很易与植物繁殖器官接触而影响 有性生殖过程。为避免这种不利影响, 需对套网处 理进行“升级”。如若隔绝的传粉者为鸟类, 则仅需 使用支撑较好的细铁丝网即可(如Fang et al, 2012); 如果使用尼龙网袋或纱袋隔绝传粉昆虫, 最好使用 硬铁丝等制作支架支撑网袋，并在封口处用绳线或 细铁丝密封(见《生物多样性》2007年第 15 卷第6期 封面)。此外, 网袋的表面积较大可能富集空气中的 花粉, 使得套网组接受比自然组更多的风媒花粉; 相反的情况, 尽管网袋孔径大于花粉直径, 网袋在 某种程度上仍有可能阻挡风媒花粉的自由通行。因 此, 为验证套袋对花粉的富集或阻碍, 需在套袋内 外设置黏性玻片比较花粉密度, 以判断是否受到该 类因素的影响(Sacchi \& Price, 1988)。

其二为动物因素。套网往往无法隔绝某些小型 昆虫的访问而影响实验结果。使用更小网孔的网袋 或多层套网可以增加对小型昆虫访问的阻碍, 但也 可能影响风媒花粉的进入。Baker和Cruden (1991)使 用杀虫剂喷酒植物以驱除蓟马和蚜虫并取得了不错 的效果。此外, 食花者(florivore)和食果者(frugivore) 破坏花果可能影响有效数据的获取, 研究者应选择 适当手段, 如增加套网时间等进行规避。

其三为植物因素。植物复杂的性系统(张大勇, 2004)、交配系统(陈小勇, 2004)和传粉系统(黄双全 和郭友好, 2000)可能增加实验结果解读的复杂性。 
研究者需在套网的基础上增加套袋(同时隔绝风媒 和虫媒)和去雄处理并进行组合, 判断自动自交 (autogamy)和无融合生殖 (apomixis) 在风媒传粉植 物中发生的可能性(技术路线详见图1)。

\section{3 空气动力学}

风媒传粉研究往往受诸多实际问题的限制, 如 人力成本制约大量黏性玻片的花粉计数, 时间成本 制约传粉者排除法的野外开展, 因为从套网处理到 结实率数据获取往往需要等候太长时间。在这种情 况下, 空气动力学则是一个很好的替代方法。研究 者只需在野外快速收集研究物种的组织材料, 通过 干燥或冷冻等方法保存后带回实验室, 开展后续实 验即可。想在实验室内完全理解花粉在空气中的运 动规律和实际效果是十分困难的, 因为太多的变量 需要考虑(见Ackerman \& Kevan, 2005)。但就一般研 究而言, 风媒传粉效率主要受花粉终端沉降速度 (terminal settling velocity, $U_{\mathrm{t}}$ ) 和周围气流加速场两 个因素的影响(Niklas, 1985)。 $U_{\mathrm{t}}$ 可用来推测植物风 媒传粉的可能性, 风洞(wind tunnel)实验可以反映 雌荵周围气流加速场中花粉的真实运动情况, 而电 脑模拟可以对其进行计算和评估(Niklas \& Buchmann, 1987; Bolinder et al, 2015)。

\subsection{1 花粉沉降系数}

$U_{\mathrm{t}}$ 就是在静止的气流中, 花粉垂直下落达到稳 定状态, 即重力和阻力平衡后的运动速度(Niklas \& Spatz, 2012), 风媒传粉植物的 $U_{\mathrm{t}}$ 一般为 $2-6 \mathrm{~cm} / \mathrm{s}$ (郭
友好, 1994)。密度低、体积小的花粉往往 $U_{\mathrm{t}}$ 较小, 因 而具有更大的传播距离(Cresswell et al, 2010)。以下 介绍测量 $U_{t}$ 的两种常用方法及使用中的问题和改进 建议:

(1)频闪摄影法。包括黑箱子和玻璃圆筒法，二 者原理相似, 只是提供花粉在静止气流中下落的装 置不同：前者使用内壁涂有亚光材料以减少光散射 的黑箱子(Niklas \& Buchmann, 1987), 后者使用圆 柱形的玻璃圆筒(Bolinder et al, 2015)。测定技术均 采用静止流体中花粉下落的频闪摄影技术。实验时 需在黑暗的环境中放置好闪光灯和相机镜头, 当闪 光灯和相机快门打开的同时释放花粉, 之后捕捉花 粉的运动图像。使用匀速下落过程中两张连续图像 的间距乘以闪光频率便可求得 $U_{\mathrm{t}}$ 。 Bolinder等(2015) 使用玻璃圆筒法测得Ephedra foeminea 的 $U_{\mathrm{t}}$ 在4.7$21.44 \mathrm{~cm} / \mathrm{s}$ 之间, 这种大的差异是由花粉的不同聚 集程度引起的。Hirose和Osada (2016)的玻璃圆筒法 也大致类似，只不过在玻璃圆筒底端安装发射波长 为635 $\mathrm{nm}$ 的激光装置, 替代闪光灯照明以协助花粉 运动过程的拍摄; 他们研究的 5 种植物的 $U_{\mathrm{t}}$ 平均值分 布在1.03-3.2 cm/s之间, 花粉越大的物种, $U_{\mathrm{t}}$ 值越大。

(2)沉降塔法。沉降塔设备主要包括4个部分(详 见Di-Giovanni et al, 1995; Borrell, 2012): 花粉释放 装置、双层圆柱筒(提供花粉下落所需的静止空气环 境)、转盘(其上装载径向排列的单面涂有凡士林的 玻片, 并与花粉的释放同步进行)、控制面板(电动机

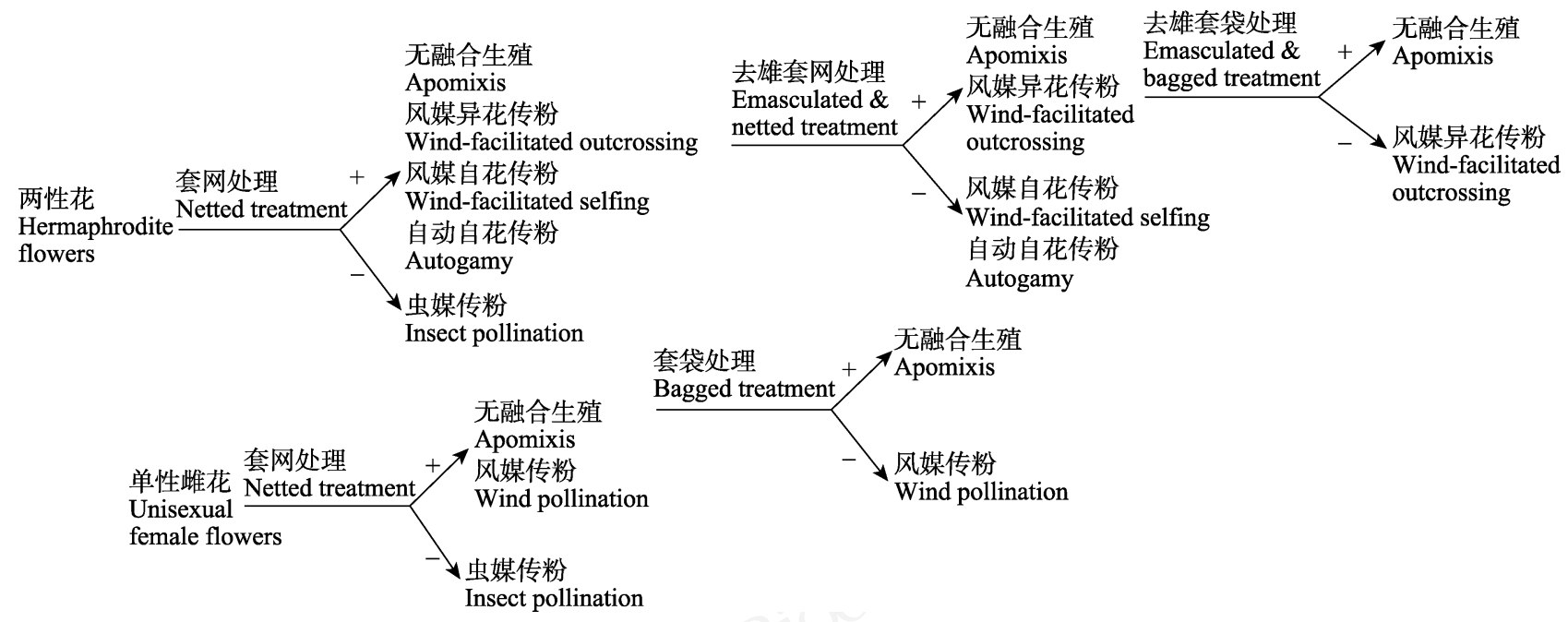

图 1 传粉者排除法结合传粉处理检验风媒传粉的技术路线。+表示结籽率 $>\mathbf{0},-$ 表示结籽率 $=\mathbf{0}$ 。

Fig. 1 Technical routes to test wind pollination by pollinator exclusion and pollination treatments. + indicates seed set $>0$, - indicates seed set $=0$. 
供电, 可以控制圆盘转速)。实验中, 花粉垂直下落 距离已知, 根据花粉在转盘玻片上的沉积位置和转

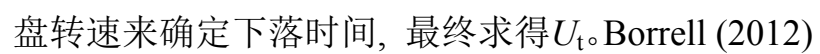
对Di-Giovanni等(1995)的沉降塔设备加以改进, 使 用小型的振动刷代替原来的充气装置释放花粉, 对 6 种禾本科和 4 种雪松属 $(C e d r u s)$ 植物的 $U_{\mathrm{t}}$ 进行快速 测定; 发现不同物种 $U_{\mathrm{t}}$ 差异显著, 禾本科植物的 $U_{\mathrm{t}}$ 平均值在3.81-5.29 cm/s之间, 显著低于雪松属植物 的 $U_{\mathrm{t}}$ (6.72-9.18)。作者预测这种差异可以影响花粉 的传播距离进而可能影响不同植物的遗传多样性 和居群结构。

(3) 问题和改进建议。为保证精确性, $U_{\mathrm{t}}$ 的测定 最好采用新鲜或者冻存保鲜的花粉(Karl Niklas, 私 人联系)。但对于一些野外采集后难以保鲜的花粉, 终端沉降速度只能在野外实时测定。例如买麻藤 (Gnetum montanum) 的花药一旦离体或稍用力碰触 便会氧化发黑, 即使迅速 $-20^{\circ} \mathrm{C}$ 冻存也无法避免(朱 亚如, 个人观察)。另外, 沉降塔设备在野外使用不 便, Niklas和Buchmann (1987)的黑箱子法更加便捷。 但在实际使用过程中, 我们发现闪光灯的效果并不 理想, 而强光手电的效果更佳。可预先在黑箱内壁 制作一些刻度条背景, 便于记录花粉下落的距离。 使用高清画质单反相机连续摄像, 记录花粉匀速下 落的距离和时间, 即可求得 $U_{\mathrm{t}}$ 。

\subsection{2 风洞实验}

关于风洞实验，其工作场的建立需满足以下 3 个条件: (1)配备变速风扇。Niklas和Buchmann (1987) 使用变速范围为 $0.20-15.5 \mathrm{~m} / \mathrm{s}$ 的风扇; (2)工作场中 气流的稳定性。风洞中放置充满氦气的中性漂浮球, 通过频闪照明观察球排出风洞的轨迹来判断 (Niklas, 1984)。因闪光频率已知, 该技术还可用来 校准气流速度。实验中, 也可借助风速仪对气流速 度进行周期性检测; (3)相对均匀的花粉浓度。实验 中释放的花粉量可由体积判断, 释放点在风洞内部, 花粉浓度可通过连续监测穿过拍摄区的花粉数量 判断。Niklas和Buchmann (1987)对2种麻黄(Ephedra trifurca和E. nevadensis) 的风洞实验结果显示, 某一 特定的风速下, 植物在传粉过程中更易接受同种花 粉。Cresswell等(2007)使用新鲜的花粉和雌球花材 料, 在风速和花粉密度均稳定的风洞中, 对 3 种松 科植物不同部位的花粉接受能力进行了比较, 结果 发现3种植物的迎风面均能接受约 $70 \%$ 的花粉, 背
风面只能接受约 $2 \%$ 的花粉, 而左右侧面接受的花 粉比例在 2 种松属 (Pinus) 植物中无差异, 但在 Cedrus libani 中却差异显著。

\subsection{3 电脑模拟}

基于风洞实验测定的不同物种花粉运动的差 异, 可通过电脑模拟来评估导致这种空气动力学差 异的物理基础(Niklas \& Buchmann, 1987; Cresswell et al, 2007)。电脑模拟前需获取的指标有: (1)花粉的 密度、大小; (2)雌性生殖器官的大小、形态和普遍 的朝向; (3)平均风速(Bolinder et al, 2015)。花粉密度 测定可使用密度梯度离心法(Forster \& Flenley, 1993), 但如果 $U_{\mathrm{t}}$ 已知, 也可以采用以下公式:

$$
\rho=\frac{9 U_{\mathrm{t}} \mu}{2 r^{2} \mathrm{~g}}+\rho_{0}
$$

其中 $r$ 是花粉半径, $\mathrm{g}$ 是重力加速度, $\rho$ 和 $\rho_{0}$ 分别是花 粉和介质的密度, $\mu$ 是空气的动力粘度系数, $U_{\mathrm{t}}$ 是终 端沉降速度(Niklas，1985); 而雌性生殖器官的形态 和大小可根据实际情况使用数码拍照、MicroCT扫 描仪或扫描电镜测定(Cresswell et al, 2007)。雌性生 殖器官周围花粉行为的模拟详见Niklas和Kerchner (1986)设计的程序。Niklas和Buchmann (1987)使用 电脑模拟 E. trifurca和E. nevadensis肧珠周围气流场 中花粉的运动行为, 得到了与风洞实验相似的结果; 并且预测, 不同物种花粉大小和密度的差异, 导致 各物种花粉具有特定的惯性特征, 结合不同物种胚 珠所形成的特异空气动力微环境, 即产生了风媒传 粉过程中更易接受同种花粉的现象。Bolinder等 (2015)的电脑模拟发现 E. foeminea不具备任何风媒 花粉捕捉的功能特征, 这也与其虫媒传粉的早期观 点一致。

\section{3 风媒传粉影响因素的研究方法}

传粉生物学的发展, 已从单纯描述传粉现象向 揭示传粉机理和传粉选择作用下植物的适应性进 化等方向发展。因此, 需对影响风媒传粉的因素进 行研究, 以更深入理解风媒传粉发生的原因和后 果。影响风媒的因素很多, 以下将其分为非生物和 生物两大类, 就其部分重要因素进行论述, 更全面 的因素总结见表1。

\section{1 非生物因素}

研究非生物因素，一般须在不同环境条件下， 
在测量风媒传粉效率的基础上，对某个或几个关注 的非生物因素进行测量(见表1), 以寻找其中关联。 风速、降雨量、温湿度等基础数据可从附近的气象 站、野外工作台站等获取; 但如果想了解实验居群 微环境中的相关数据, 则须针对性购买相关仪器进 行测量。如我们购买的FODY TEMPUS Pro小型气 象站, 能够连续监测大部分影响风媒传粉的非生物 因素。

风速是影响风媒传粉的一个重要因素, 不但影 响花粉的散播距离, 同时还影响柱头对花粉的捕捉 (Whitehead, 1969)。当风速大到可以克服花粉的 $U_{\mathrm{t}}$, 但又不至于阻止柱头对花粉的接收时，同种个体间 的花粉传递效率最高(Niklas，1985; Culley et al, 2002)。鲁先文等(2008)使用风速计和黏性玻片法检 验风速对沙棘(Hippophae rhamnoides) 风媒传粉距 离的影响, 发现风速较低时花粉传播距离一般不超 过 $25 \mathrm{~m}$; 但风速超过 $3.5 \mathrm{~m} / \mathrm{s}$ 时, 顺风花粉传播距离 可高至 85 m。Molina等(2001)使用Burkard定量空气 自动取样器研究车前属(Plantago) 植物花粉密度与 环境因素的关系, 发现温度、湿度和风速对空气中 的花粉密度均有显著影响。

高降雨量带来的湿度增加可影响花粉的聚集 程度, 风媒花粉在潮湿空气中极易变黏而导致 $U_{t}$ 增 加; 湿度还能影响花药的脱水程度, 从且影响花粉 的释放(Niklas, 1985)。降雨最直接的影响就是“雨滴 清除” 现象, 即适合大小的雨滴可在较短时间内清 除空气中所有的花粉 (McDonald，1962)。Gong等 (2016)在晴雨两种天气条件于小叶买麻藤(Gnetum parvifolium)雌雄株附近悬挂黏性玻片, 发现晴天时, 雄䧳株附近玻片均能接收到花粉, 但雨天时雌株附 近玻片上却无花粉捕获。

\section{2 生物因素}

花粉的大小、密度、形态和超微结构都能影响 风媒传粉效率，风媒花粉的大小一般在 20-40 $\mu \mathrm{m}$, 表面平滑干燥以减少花粉聚集(Ackerman \& Kevan, 2005), 一些风媒物种的花粉壁内部存在大量空白 区域，有利于增加其浮力(Wodehouse，1935)。利用 电镜技术就能清楚观察到花粉表面和内部结构差 异(Tekleva，2016)。Bolinder等(2015)利用扫描和透 射电镜技术对麻黄属植物的花粉进行研究, 发现花 粉壁的超微结构与终端沉降速度是相关联的, 花粉 壁顶盖下层空间内颗粒物质的相对比例和数量共
同决定花粉密度, 并与整体的大小一起决定 $U_{\mathrm{t}}$, 进 而影响花粉散播能力。

而花柱的结构也十分重要, 花柱暴露性、柱头 黏性和表面积的增加都有利于提高风媒花粉捕获 效率，如裸子植物能分泌传粉滴、禾本科植物具羽 毛状或帚刷状柱头等(Faegri \& van der Pijl, 1979)。 朱俊义等(2014)利用扫描电镜技术, 发现部分桦木 科植物的花柱表皮细胞能形成乳突，有利于增加花 粉接受面积从而提高风媒传粉效率。

除以上花部构成因素外, 开花式样也能对风媒 传粉产生重要影响(Friedman \& Barrett, 2009)。一般 来讲, 风速随着高度的增加而增加, 对于风媒传粉 的雌雄同株物种，雄花处于较高位置更有利于花粉 的散播(Bickel \& Freeman, 1993)。Niklas (1987)利用 风洞实验，发现紧凑和弥散型圆雉花序在花粉接受 方面具二态性, 即紧凑型圆雉花序对气流的阻力较 大，所以花粉在花序的上风向处偏离，而在下风向 处通过沉降被捕捉; 而弥散型圆雉花序对气流的阻 力较小, 因此大部分的花粉在上风向处与花序碰撞 并被捕捉。Friedman和Harder (2004)采用花序捆绑 固定法以减少花序的随风移动幅度, 发现该处理降 低了紧凑型圆雉花序的花粉移出量，但不影响柱头 花粉接受量; 而在弥散型花序中, 花粉的移出量不 受影响, 但柱头花粉接受量却显著降低。

\section{4 结语}

在自然界中，仅根据风媒传粉综合征(wind pollination syndrome; 表1; Faegri \& van der Pijl, 1979)来判断风媒传粉的有无是不全面的, 有些植 物即使不完全具备这些典型特征，也可能具有风媒 传粉模式(Gong et al, 2016)。在全球气候变化、生境 片断化、生物入侵等自然选择压力下，植物某些细 微特征的改变, 都有可能带来传粉模式的转变 (Friedman, 2011)。

相对于动物传粉研究, 目前我国风媒传粉研究 所关注的植物种类仍十分有限。已发表的论文大多 仅描述风媒植物的适应特征(如朱俊义等, 2014), 或 使用野外实验方法研究风媒传粉的影响因素和生 殖贡献(如鲁先文等，2008; 崔大练等，2008), 目前 仍未见空气动力学方法在我国风媒传粉研究中的 应用。此外, 仅有少量研究关注风媒与动物媒的并 存现象及传粉转变的适应意义(Huang et al, 2013; 
Gong et al, 2016; Wang et al, 2016)。

风媒传递的花粉往往体积较小而肉眼难辨, 相 对于可直接观察的动物传粉过程, 其在野外研究中 的隐蔽性更强。在野外实验中, 研究者应首选使用 “花粉捕获法+传粉者排除法”的组合方法, 明确风 媒传粉的可能性和生殖贡献大小。野外时间充足时, 应在各种环境条件下使用该组合方法对比测量风 媒传粉效率。如野外条件受限, 研究者应快速收集 相关植物组织材料, 在实验室内开展空气动力学研 究。然而, 野外实验和室内方法并不能相互替代, 两类方法的综合对比运用才能由表及里地揭示自 然传粉现象和发生机理(黄双全, 2012)。同时应特别 注意, 风媒和虫媒传粉可并存于一种植物, 此类研 究应结合使用传粉昆虫行为的研究方法(龚燕兵和 黄双全, 2007)。本文就风媒传粉的研究方法进行了 相对全面的总结和评价, 希望能对我国开展相关研 究的学者有所裨益。

致谢: 感谢郭友好教授和Karl J. Niklas教授提供的 宝贵资料和建议!

\section{参考文献}

Ackerman JD (2000) Abiotic pollen and pollination: ecological, functional, and evolutionary perspectives. Plant Systematics and Evolution, 222, 167-185.

Ackerman JD, Kevan PG (2005) Abiotic pollination. In: Practical Pollination Biology (eds Dafni A, Kevan PG, Husband BC), pp. 435-480. Enviroquest Ltd., Cambridge.

Baker JD, Cruden RW (1991) Thrips-mediated self-pollination of two facultatively xenogamous wetland species. American Journal of Botany, 78, 959-963.

Bawa KS (1990) Plant-pollinator interactions in tropical rain forests. Annual Review of Ecology and Systematics, 21, 399-422.

Bickel AM, Freeman DC (1993) Effects of pollen vector and plant geometry on floral sex ratio in monoecious plants. American Midland Naturalist, 130, 239-247.

Bolinder K, Niklas KJ, Rydin C (2015) Aerodynamics and pollen ultrastructure in Ephedra. American Journal of Botany, 102, 457-470.

Borrell JS (2012) Rapid assessment protocol for pollen settling velocity: implications for habitat fragmentation. Bioscience Horizons, 5, 1-9.

Chen XY (2004) Mating systems. In: Plant Life-History Evolution and Reproductive Ecology (ed. Zhang DY), pp. 258-279. Science Press, Beijing. (in Chinese) [陈小勇
(2004) 交配系统. 见: 植物生活史进化与繁殖生态学 (张大勇主编), 258-279页. 科学出版社, 北京.]

Cox PA (1991) Abiotic pollination: an evolutionary escape for animal-pollinated angiosperms. Philosophical Transactions of the Royal Society B: Biological Sciences, 333, 217-224.

Cresswell JE, Henning K, Pennel C, Lahoubi M, Patrick MA, Young PG, Tabor GR (2007) Conifer ovulate cones accumulate pollen principally by simple impaction. Proceedings of the National Academy of Sciences, USA, 104, 18141-18144.

Cresswell JE, Krick J, Patrick MA, Lahoubi M (2010) The aerodynamics and efficiency of wind pollination in grasses. Functional Ecology, 24, 706-713.

Cui DL, Man XL, Ma YX, Zhang YS (2008) Study on pollination ecology of Paris verticillata M.-Bieb. Acta Botanica Boreali-Occidentalia Sinica, 28, 298-302. (in Chinese with English abstract) [崔大练, 满秀玲, 马玉心, 张玉生 (2008) 北重楼传粉生态学研究. 西北植物学报, 28, 298-302.]

Culley TM, Weller SG, Sakai AK (2002) The evolution of wind pollination in angiosperms. Trends in Ecology \& Evolution, 17, 361-369.

Dafni A, Dukas R (1986) Insect and wind pollination in Urginea maritima (Liliaceae). Plant Systematics and Evolution, $154,1-10$.

Darwin C (1876) The Effects of Cross and Self Fertilisation in the Vegetable Kingdom. John Murray, London.

Di-Giovanni F, Kevan PG, Nasr ME (1995) The variability in settling velocities of some pollen and spores. Grana, 34, 39-44.

Dodd ME, Silvertown J, Chase MW (1999) Phylogenetic analysis of trait evolution and species diversity variation among angiosperm families. Evolution, 53, 732-744.

Elzinga JA, Atlan A, Biere A, Gigord L, Weis AE, Bernasconi $G$ (2007) Time after time: flowering phenology and biotic interactions. Trends in Ecology \& Evolution, 22, 432-439.

Faegri K, van der Pilj L (1979) The Principles of Pollination Ecology, 3rd edn. Pergamon Press, Oxford.

Fang Q, Chen YZ, Huang SQ (2012) Generalist passerine pollination of a winter-flowering fruit tree in central China. Annals of Botany, 109, 379-384.

Forster M, Flenley JR (1993) Pollen purification and fractionation by equilibrium density gradient centrifugation. Palynology, 17, 137-155.

Frenz DA (1999) Comparing pollen and spore counts collected with the Rotorod Sampler and Burkard spore trap. Annals of Allergy, Asthma \& Immunology, 83, 341-349.

Friedman J (2011) Gone with the wind: understanding evolutionary transitions between wind and animal pollination in the angiosperms. New Phytologist, 191, 911-913.

Friedman J, Barrett SCH (2009) Wind of change: new insights on the ecology and evolution of pollination and mating in wind-pollinated plants. Annals of Botany, 103, 1515-1527. 
Friedman J, Harder LD (2004) Inflorescence architecture and wind pollination in six grass species. Functional Ecology, $18,851-860$.

Gong YB, Huang SQ (2007) On methodology of foraging behavior of pollinating insects. Biodiversity Science, 15, 576-583. (in Chinese with English abstract) [龚燕兵, 黄双 全 (2007) 传粉昆虫行为的研究方法探讨. 生物多样性, 15, 576-583.]

Gong YB, Yang M, Vamosi JC, Yang HM, Mu WX, Li JK, Wan T (2016) Wind or insect pollination? Ambophily in a subtropical gymnosperm Gnetum parvifolium (Gnetales). Plant Species Biology, 31, 272-279.

Guo YH (1994) Pollination biology and the evolution of plants. In: Plant Evolutionary Biology (eds Chen JK, Yang J), pp. 232-280. Wuhan University Press, Wuhan. (in Chinese) [ 郭 友好 (1994) 传粉生物学与植物的进化. 见: 植物进化生 物学 (陈家宽, 杨继主编), 232-280页. 武汉大学出版社, 武汉.]

Hegland SJ, Nielsen A, Lázaro A, Bjerknes AL, Totland Ø (2009) How does climate warming affect plant-pollinator interactions? Ecology Letters, 12, 184-195.

Hirose Y, Osada K (2016) Terminal settling velocity and physical properties of pollen grains in still air. Aerobiologia, 32, 385-394.

Huang SQ (2012) Pollination biology in China in the 21st century: getting a good start. Biodiversity Science, 20, 239-240. (in Chinese) [黄双全 (2012) 二十一世纪中国传粉生物学 的研究: 良好的开端. 生物多样性, 20, 239-240.]

Huang SQ, Guo YH (2000) Advances in pollination biology. Chinese Science Bulletin, 45, 225-237. (in Chinese) [黄双 全, 郭友好 (2000) 传粉生物学的研究进展. 科学通报, 45, 225-237.]

Huang SQ, Xiong YZ, Barrett SCH (2013) Experimental evidence of insect pollination in Juncaceae, a primarily wind-pollinated family. International Journal of Plant Sciences, 174, 1219-1228.

Ickert-Bond SM, Renner SS (2016) The Gnetales: recent insights on their morphology, reproductive biology, chromosome numbers, biogeography, and divergence times. Journal of Systematics and Evolution, 54, 1-16.

Jin B, Zhang L, Lu Y, Wang D, Jiang XX, Zhang M, Wang L (2012) The mechanism of pollination drop withdrawal in Ginkgo biloba L. BMC Plant Biology, 12, 59.

Kato M, Inoue T (1994) Origin of insect pollination. Nature, 368,195

Kearns CA, Inouye DW (1993) Techniques for Pollination Biologists. University Press of Colorado, Niwot.

Kono M, Tobe H (2007) Is Cycas revoluta (Cycadaceae) windor insect-pollinated? American Journal of Botany, 94, $847-855$

Linder HP (1998) Morphology and the evolution of wind pollination. In: Reproductive Biology in Systematics, Conservation and Economic Botany (eds Owens SJ, Rudall PJ), pp.
123-135. Royal Botanic Gardens, Kew.

$\mathrm{Lu}$ XW, Ma RJ, Sun K (2008) Determination of the wind pollination distances and flowering characteristics of Hippophae rhamnoides L. ssp. sinensis Rousi (Elaeagnaceae). Acta Ecologica Sinica, 28, 2518-2525. (in Chinese with English abstract) [鲁先文, 马瑞君, 孙坤 (2008) 中国沙 棘(Hippophae rhamnoides L. ssp. sinensis Rousi)的开花特 性及风媒传粉距离的检测. 生态学报, 28, 2518-2525.]

McDonald JE (1962) Collection and washout of airborne pollens and spores by raindrops. Science, 135, 435-437.

Molina RT, Palacios IS, RodrÍguez AFM, Muñoz JT, Corchero AM (2001) Environmental factors affecting airborne pollen concentration in anemophilous species of Plantago. Annals of Botany, 87, 1-8.

Niklas KJ (1984) The motion of windborne pollen grains around conifer ovulate cones: implications on wind pollination. American Journal of Botany, 71, 356-374.

Niklas KJ (1985) The aerodynamics of wind pollination. The Botanical Review, 51, 328-386.

Niklas KJ (1987) Pollen capture and wind-induced movement of compact and diffuse grass panicles: implications for pollination efficiency. American Journal of Botany, 74, 74-89.

Niklas KJ (2015) A biophysical perspective on the pollination biology of Ephedra nevadensis and E. trifurca. The Botanical Review, 81, 28-41.

Niklas KJ, Buchmann SL (1987) Aerodynamics of pollen capture in two sympatric Ephedra species. Evolution, 41, 104-123.

Niklas KJ, Kerchner V (1986) Aerodynamics of Ephedra trifurca. II. Computer modelling of pollination efficiencies. Journal of Mathematical Biology, 24, 1-24.

Niklas KJ, Spatz HC (2012) Plant Physics. University of Chicago Press, Chicago.

Ollerton J, Erenler H, Edwards M, Crockett R (2014) Extinctions of aculeate pollinators in Britain and the role of large-scale agricultural changes. Science, 346, 1360-1362.

Owens JN, Takaso T, Runions CJ (1998) Pollination in conifers. Trends in Plant Science, 3, 479-485.

Peng DL, Zhang ZQ, Niu Y, Yang Y, Song B, Sun H, Li ZM (2012) Advances in the studies of reproductive strategies of alpine plants. Biodiversity Science, 20, 286-299. (in Chinese with English abstract) [彭德力, 张志强, 牛洋, 杨扬, 宋波, 孙航，李志敏 (2012) 高山植物繁殖策略的研究进 展. 生物多样性, 20, 286-299.]

Regal PJ (1982) Pollination by wind and animals: ecology of geographic patterns. Annual Review of Ecology and Systematics, 13, 497-524.

Sacchi CF, Price PW (1988) Pollination of the arroyo willow, Salix lasiolepis: role of insects and wind. American Journal of Botany, 75, 1387-1393.

Tekleva M (2016) Pollen morphology and ultrastructure of several Gnetum species: an electron microscopic study. Plant Systematics and Evolution, 302, 291-303. 
Vamosi JC, Knight TM, Steets JA, Mazer SJ, Burd M, Ashman TL (2006) Pollination decays in biodiversity hotspots. Proceedings of the National Academy of Sciences, USA, 103, 956-961.

Wang LL, Zhang C, Yang ML, Zhang GP, Zhang ZQ, Yang YP, Duan YW (2016) Intensified wind pollination mediated by pollen dimorphism after range expansion in an ambophilous biennial Aconitum gymnandrum. Ecology and Evolution, 7, 541-549.

Wang Q, Li CL, Yang SY, Huang R, Chen FL (1997) Pollination biology of Cycas panzhihuaensis L. Zhou et S. Y. Yang. Acta Botanica Sinica, 39, 156-163. (in Chinese with English abstract) [王乾, 李朝軹, 杨思源, 黄荣, 陈发林 (1997) 攀枝花苏铁传粉生物学研究. 植物学报, 39, 156-163.]

Wang XQ, Ran JH (2014) Evolution and biogeography of gymnosperms. Molecular Phylogenetics and Evolution, 75, 24-40.

Wetschnig W, Depisch B (1999) Pollination biology of Welwitschia mirabilis HOOK. f. (Welwitschiaceae, Gnetop- sida). Phyton, 39, 167-183.

Whitehead DR (1969) Wind pollination in the angiosperms: evolutionary and environmental considerations. Evolution, $23,28-35$.

Wodehouse RP (1935) Pollen Grains: Their Structure, Identification and Significance in Science and Medicine. McGrawHill, New York.

Zhang DY (2004) Plant Life-History Evolution and Reproductive Ecology. Science Press, Beijing. (in Chinese) [张大勇 (2004) 植物生活史进化与繁殖生态学. 科学出版社, 北 京.]

Zhu JY, Zhang LF, Shen P, Ren BQ, Liang Y, Chen ZD (2014) Wind pollination characteristics of styles in Betulaceae. Chinese Bulletin of Botany, 49, 524-538. (in Chinese with English abstract) [朱俊义, 张力凡, 沈鹏, 任保青, 梁宇, 陈之端 (2014) 桦木科植物花柱适应风媒传粉的特征. 植物学报, 49, 524-538.]

(责任编委：黄双全 责任编辑：时意专) 